\title{
Histoire et courants architecturaux du bâtiment scolaire, une nouvelle ère à venir?
}

History and architectural currents of the school building, a new era to come?

Sarah Barthelemy et Laurent Jeannin

\author{
(2) OpenEdition \\ Journals \\ Édition électronique \\ URL : https://journals.openedition.org/trema/5416 \\ DOI : 10.4000/trema.5416 \\ ISSN : 2107-0997 \\ Éditeur \\ Faculté d'Éducation de l'université de Montpellier
}

Référence électronique

Sarah Barthelemy et Laurent Jeannin, « Histoire et courants architecturaux du bâtiment scolaire, une nouvelle ère à venir? », Tréma [En ligne], 52 | 2019, mis en ligne le 01 septembre 2019, consulté le 16 janvier 2023. URL : http://journals.openedition.org/trema/5416; DOI : https://doi.org/10.4000/trema. 5416

Ce document a été généré automatiquement le 16 janvier 2023.

Tous droits réservés 


\section{Histoire et courants architecturaux du bâtiment scolaire, une nouvelle ère à venir ?}

History and architectural currents of the school building, a new era to come?

Sarah Barthelemy et Laurent Jeannin

\section{Introduction}

1 Au cours de l'histoire, les bâtiments et les villes ont reflété les différents courants et les enjeux de la société. Il en va de même pour l'architecture scolaire, équipement particulièrement important à partir du début du $19^{\text {ème }}$ siècle, lorsque le gouvernement impose aux communes la mise à disposition d'un espace dédié pour les instituteurs (Durandet, 2015).

2 Nous nous proposons de revoir l'histoire de l'architecture scolaire sous le prisme du bâti et de ses caractéristiques, de manière à identifier des liens entre enjeux de société, architecture et caractéristiques pédagogiques.

\section{Une architecture scolaire « normée »}

\section{Architecture / pédagogie}

\section{Enseignement mutuel et enseignement confessionnel}

3 La notion d'architecture scolaire apparait en tant que telle au début du $19^{\text {ème }}$ siècle, avec l'avènement des maisons d'école sous la loi Guizot et l'apparition de guides de référence sur la construction des écoles. Deux méthodes d'enseignement sont alors en place en France (Châtelet, 1999 ; Lainé, 1996) : d'un côté le mode simultané, aussi appelé enseignement confessionnel puisqu'il est majoritairement donné par l'Église; d'un autre le mode mutuel, importé d'Angleterre et bousculant les codes alors établis de 
l'enseignement confessionnel. Ces deux méthodes sont liées à des espaces d'apprentissage conçus pour elles, normés, décrits par des commissions d'architectes nommées (Châtelet, 2008).

Les modes mutuel et confessionnel ont des besoins différents, qui se retranscrivent dans les bâtiments de ces périodes. Le mode mutuel est basé sur le travail autonome des élèves avec une supervision des enseignants, tandis que le mode simultané oriente l'enseignement sur l'écoute attentive du maitre. Par conséquent, les écoles mutuelles peuvent accueillir plus d'une centaine d'élèves en même temps (voire d'avantage), tandis que les écoles confessionnelles s'organisent autour de groupes de trente à cinquante élèves au maximum. En miroir, deux typologies de bâtiments ont été conçus pendant cette période :d'une part les grande halles de $20 \mathrm{~m}$ de long par $10 \mathrm{~m}$ de large et $6 \mathrm{~m}$ de hauteur sous plafond, constituée de rangs d'une dizaine d'élèves avec des présentoirs à chaque extrémité et une estrade avec les bureaux des enseignants sur un côté de la halle, ont été construites pour l'enseignement mutuel (Durandet, 2014); d'autre part, des salles d'environ $50 \mathrm{~m} 2$, avec $4 \mathrm{~m}$ de hauteur sous plafond et des tables et chaises fixes en rang devant une estrade et un tableau, ont été pensées pour le mode simultané (Lainé, 1996). Avec la disparition du mode mutuel en 1850, seules sont restées les écoles simultanées, dont la structure n'a pas beaucoup évolué depuis lors.

5 Dès cette période, les maisons d'école sont conçues selon des logiques d'hygiène et de sécurité. L'école est vue comme un sanctuaire, qui protège les élèves de l'extérieur et de ses vicissitudes. Elle propose également un espace dans lequel on peut se dépenser et prendre l'air (préau, cour).

6 Au même moment, les architectures destinées aux lycées sont conçues selon un autre principe. Créés en 1802, avec la volonté d'en faire des monuments durables (Le Cœur, 2005), les lycées suivent des conditions minimales et plusieurs règles concernant les travaux de réaménagement d'anciens collèges ou bâtiments congréganistes devenus biens nationaux, selon la loi établie par Fourcroy. N'ayant que peu de recul sur ce qu'est le lycée et peu d'informations concernant l'architecture scolaire, Fourcroy va inventer seul cette nouvelle entité, en s'appuyant sur les groupes "classes», "en tant qu'ensembles homogènes et gradués d'élèves » (Le Cœur, 2005) autour desquelles vont s'organiser les bâtiments. A chaque classe correspond alors une salle de cours, une salle d'étude et un dortoir d'une trentaine de lits. Les salles de cours sont regroupées au rezde-chaussée tandis que les salles d'études se situent dans les étages, au même titre que les dortoirs. Enfin, l'établissement doit contenir «des cours spacieuses pour les récréations et quelques parties couvertes d'arbres » (Le Coeur, 2005). Une typologie architecturale va être définie en 1843 suite aux nombreux problèmes de mise en œuvre des lycées dans des bâtiments existants. Le texte du ministre Villemain questionne pour la première fois la place du lycée dans son contexte urbain, et la notion d'environnement des lycées (Le Cœur, 2005). Les élèves sont alors groupés en classes, mais aussi en sections (petits, moyens et grands) et répartis dans des quartiers spécifiques, disposant chacun d'une cour qui leur est propre.

7 Tout comme pour les maisons d'école, l'hygiène et la propreté sont au centre des réflexions. A partir de 1860, une grande enquête est menée pour anticiper les réformes. Gustave Rouland, premier ministre de l'instruction publique, considère alors les bâtiments dans leur ensemble et sensibilise les recteurs, inspecteurs et proviseurs à ces questions. Il constitue un cadre administratif qui touche aux questions d'architecture et s'attache ainsi une commission «chargée de donner son avis, tant sur les plans et 
devis concernant l'établissement des nouveaux lycées, que sur les réparations, appropriations ou agrandissements des bâtiments affectés aux anciens lycées " (Circulaire du 20 décembre 1861, citée par Le Cœur, 2005). Cette commission, constituée de quatre architectes, se voit chargée de rédiger un projet de programme à l'attention des maitrises d'ouvrage, mais également des maitrises d'œuvre qui y trouveront «les indications et les idées générales dont ils ont besoin pour préparer leurs plans " (Circulaire du 20 décembre 1861, citée par Le Cœur, 2005). Ces idées se soucient pour la première fois du confort des enfants et du point de vue des familles, évoquant la lumière, l'espace et l'air. Gustave Rouland indique que «si nous pouvons faire aimer à nos jeunes élèves les lieux où se passent leurs premières années, nous auront beaucoup fait pour leur véritable éducation " (Circulaire ministérielle du 23 janvier 1860, citée par Le Cœur, 2005).

Une seule autre période dans l'histoire de l'architecture scolaire fait expressément référence à la pédagogie, et aux besoins en espace qui y sont liés. Il s'agit de la décennie 1970-1980, durant laquelle de nombreuses expérimentations autour du bâti scolaire, comme préfigurateur d'une nouvelle pédagogie ou au contraire en support d'une pédagogie existante, ont été conduites.

\section{Années 70}

9 Le début des années 70 marque une nouvelle période où les architectes et les maitres d'ouvrage cherchent à faire évoluer les pratiques par le bâti (Foster, 2004). En effet, le nombre croissant d'élèves d'origine sociale diverse, et les pratiques répandues, comme le cours magistral, montrant leurs limites face à des classes très hétérogènes, l'échec scolaire est de plus en plus problématique. "Ce qui est donc rapidement mis en question c'est l'inadaptation d'un système d'enseignement en direction d'un public scolaire de plus en plus large, dont les parents sont progressivement plus attentifs à une réussite par l'école. » (Navarro, 2012, p. 42)

L'architecture de la reconstruction est vécue comme inhumaine par les individus, qui réagissent mal aux villes dortoirs et aux immeubles en béton hors d'échelle (Knittel \& Castets-Fontaine, 2015). De nouvelles pratiques se développent, avec l'idée que l'élève doit apprendre par lui-même. Ces pratiques ont un impact sur les aménagements, la posture de l'enseignant n'étant plus centrale, et les élèves pouvant se déplacer (Jeannin, 2017). Plusieurs expériences sont instaurées, notamment à Paris, pour modifier les pratiques pédagogiques et solutionner l'échec scolaire (Foster, 2004). L'inspecteur R. Gloton est à l'origine d'une série d'expérimentations dont il reste aujourd'hui l'Ecole Vitruve dans le $20^{\mathrm{ème}}$ arrondissement de Paris. En 1974, une autre école sort de terre dans le quartier des Halles: l'école Saint-Merri. Les maitres d'ouvrage et les architectes proposent ici une école à aires ouvertes. Les Instructions Officielles précise dans une circulaire "la nécessité d'une conception modulable du bâtiment favorisant l'individualisation de l'enseignement, la pédagogie de soutien, le travail en équipe, le décloisonnement des classes et des disciplines, ainsi que l'ouverture sur le monde extérieur»(Robert, 2010, p. 70). La couleur est alors introduite dans les classes (Lainé, 1996), et les maitres-mots sont souplesse et flexibilité «qui permettent aux enseignants et aux élèves de réorganiser leur environnement comme cela leur parait souhaitable » (Willard-Magaud, 1973).

Si la pédagogie est un axe central pour dessiner les espaces scolaires, il existe d'autres leviers largement exploités au cours du développement du bâti scolaire depuis le début 
du $19^{\text {ème }}$ siècle. Dans la suite de l'article, nous nous concentrerons sur la place de l'hygiène et son impact sur les dispositifs architecturaux dans les écoles.

\section{Architecture / Hygiène}

\section{Jules Ferry, les prémices de l'hygiénisme}

L'arrivée de Jules Ferry au gouvernement constitue un tournant pour l'architecture scolaire, au primaire comme au lycée. La construction de nouveaux établissements explose dans les villes, du fait du nombre d'élèves dû à l'instruction obligatoire, et à la conjoncture sociétale de la révolution industrielle. Vingt lycées sont fondés la même année. Pour les écoles, le nombre d'élèves double en dix ans, et les constructions font de même. Il relance alors la commission des bâtiments des lycées et collèges, en même temps qu'il propose de nouveaux guides pour la construction des écoles primaires.

13 En parallèle, les prises de conscience de la fin du siècle concernant les scolioses et les myopies poussent les architectes à dessiner des bâtiments où l'éclairement et le mobilier sont repensés pour améliorer la santé des enfants (Châtelet, 1999). Les salles sont imaginées pour faire circuler l'air à travers la pièce: de grandes fenêtres sont positionnées pour apporter de la lumière d'un côté de la salle; d'autres, plus petites et en hauteur, sont situées sur le pan de mur opposé, donnant généralement dans les couloirs (Lainé, 1996). Le type de mobilier est également repensé. Il peut désormais s'adapter aux différents âges et tailles des élèves. Ce mobilier, de même que l'éclairement, conditionnent l'architecture des espaces. Les surfaces sont alors calculées selon les effectifs, avec un ratio de 1,25 à $1,50 \mathrm{~m}^{2}$ par élève, qui donne des salles de $50 \mathrm{~m}^{2}$ pour une quarantaine d'élèves. Les espaces extérieurs sont également pensés avec environ $10 \mathrm{~m}^{2}$ par élèves, ainsi que $2 \mathrm{~m}^{2}$ de préau intérieur et $5 \mathrm{~m}^{2}$ de préau couvert. On cherche également à utiliser des matériaux sains. Les préconisations vont d'ailleurs jusqu'à l'épaisseur des murs, qui ne doivent pas dépasser $45 \mathrm{~cm}$ en moellons et $35 \mathrm{~cm}$ en briques (Lainé, 1996). Préfigurant les années 20 et le courant hygiéniste, les premières mesures de Jules Ferry ont durablement impacté les aménagements des salles et l'occupation des espaces.

\section{Hygiénisme et écoles de plein air}

14 A l'aube du $20^{\text {ème }}$ siècle, les théories hygiénistes occupent le devant de la scène. L'idée est de transformer l'homme en modifiant son milieu de vie, et cela passe notamment par l'éducation des nouvelles générations, qui sont de plus en plus atteintes par le mauvais air des villes. Si l'architecture de l'école ne s'en est pas vraiment emparé en France, ces théories ont néanmoins une incidence sur les questionnements de l'architecture scolaire, auxquelles certains architectes, en collaboration avec des pédagogues, prennent part. Un comité international est créé en 1922, mais le mouvement s'essouffle après les années trente, avec l'apparition des antibiotiques (Châtelet, Lerch, Luc, 2003). En effet, les écoles de plein air sont conçues avant tout pour contrer les cas de tuberculose et protéger les enfants fragiles. Ces écoles sont pensées autour de l'accès à la nature et au soleil (Châtelet, Lerch, Luc, 2003). La première école, créée à Lyon en 1907, a pour objectif de proposer aux élèves : « double ration d'air, double ration de nourriture, demi ration de travail» (Gauthier, 1911). Un article paru en 1922, pose les principes de l'école de plein air. Elle doit se situer en périphérie des villes pour ne pas subir le mauvais air, de préférence sur un terrain 
vaste et arboré, permettant d'accéder à la nature. Le bâtiment doit s'orienter de manière à profiter du soleil, tout en se protégeant du vent et des pluies (Marchoux, 1922). «matériaux légers et peu durables, de façon à laisser, sans regrets la liberté d'aller jusqu'à abattre tout ou partie du bâtiment pour le rebâtir différemment " (Marchoux, 1922). Les principes pédagogiques sont aussi différents, avec la volonté d'apprendre aux enfants « à vivre en société, à limiter spontanément sa liberté à la liberté des autres, à compter sur soi autant que sur autrui, à mener en somme, non pas une existence tronquée d'écolier, mais la vie réelle ». La forme des salles, comme le mobilier (léger et facilement déplaçable) permettent de changer rapidement les postures passant de l'intérieur à l'extérieur ou d'une phase de travail à une phase de repos (Marchoux, 1922).

Les salles de cours sont pensées indépendantes les unes des autres, reliées par des galeries couvertes. Elles ont une capacité maximale de 30 élèves. Leurs dimensions sont de 7 mètres sur 9 et 5 mètres de hauteur sous plafond. Le sol est carrelé, et trois murs sur quatre sont vitrés, voire amovibles. Le mobilier aussi est transformé : les tables et les chaises sont individuelles, indépendantes les unes des autres, légères et adaptées aux élèves (Marchoux, 1922). devant les besoins de reconstruction massifs qui font suite à la Seconde Guerre mondiale. Les récentes études sur la qualité de l'air (Torres, Sanders \& Corsi, 2002 ; Deoux, 2010 ; Allen et al., 2016) ou l'impact de la nature (Daly, Burchette \& Torpy, 2010 ; Mozaffar \& Somayeh Mirmoradi, 2012) sur les apprentissages remettent sur le devant de la scène scientifique et politique ces préoccupations de plus d'un siècle, en regard des considérations autour de l'amiante et de la pollution des sols.

\section{Architecture / économie}

18 La période de reconstruction d'après-guerre est une période particulièrement importante dans l'histoire de l'architecture scolaire. Il faut en effet reconstruire rapidement un grand nombre d'établissements détruits, tout en absorbant les nouveaux effectifs produits par la démocratisation scolaire et le collège unique. Moins visible dans les écoles primaires, c'est une véritable explosion scolaire qui a lieu dans les collèges et les lycées. Au primaire, on enregistre une augmentation d'environ $50 \%$ des effectifs, soit 2500000 élèves (Chiffres de l'Éducation Nationale, 1980). Devant l'ampleur des besoins, le gouvernement crée une commission spéciale chargée de quantifier les besoins : environ 975 milliards d'anciens francs (23Md€) pour la période 1953-1957 (Poignant, 1960). En 1962, les établissements deviennent mixtes. Il faut donc fermer les écoles de filles et agrandir celles des garçons. La construction des établissements s'industrialise : l'État propose une série de schémas types pour aider à la construction des nouveaux bâtiments dans une circulaire de 1951. Pour accélérer les temps de conception et de construction, il organise un concours à destination des concepteurs et des constructeurs pour favoriser l'innovation dans les principes constructifs et les solutions préfabriquées. En 1964, 50 établissements sont concernés. Ce chiffre est multiplié par six, quatre ans plus tard. Ce serait plus de 2350 lycées qui ont été construits entre 1964 et 1975 (Prost, 2007). Toutefois, construction 
industrialisée ne signifie pas modèle unique. Plusieurs systèmes constructifs sont utilisés: béton armé, structures métalliques diverses ou techniques mixtes. Les éléments de plancher et de façade sont ensuite montés sur la structure. On ajoute ensuite les cloisons préparées en usine avec les portes déjà posées. Toute cette industrie repose sur une trame : $1,80 \mathrm{~m}$ puis $3,60 \mathrm{~m}$ et $7,20 \mathrm{~m}$. Cette trame est liée aux services et aux effectifs de l'établissement, qui fixent le nombre de salles, les dimensions, les localisations: "une logique d'ingénieur au service d'un programme minimum » (Prost, 2005). Le style des bâtiments est globalement issu du mouvement architectural moderne, qui privilégie des formes abstraites, blanches, formant de longs éléments de maçonnerie et de grands espaces vitrés (Lainé, 1996). Les classes sont construites en enfilade, le long d'un couloir, avec un escalier à chaque extrémité.

Plus de $50 \%$ du parc immobilier scolaire français a été construit avant la Première Guerre mondiale (explosion scolaire), et pendant la vague de reconstruction (massification). Les bâtiments dans lesquels enseignent et apprennent les usagers aujourd'hui sont donc fortement imprégnés des tendances et des caractéristiques inhérentes aux typologies que nous avons identifiées dans cet article. Les évolutions des pédagogies et des pratiques éducatives obligent à repenser ces formes d'un autre temps, pour les adapter aux enjeux actuels et de demain.

Plus de $50 \%$ du parc immobilier scolaire français a été construit avant la Première Guerre mondiale (explosion scolaire), et pendant la vague de reconstruction (massification). Les bâtiments dans lesquels enseignent et apprennent les usagers aujourd'hui sont donc fortement imprégnés des tendances et des caractéristiques inhérentes aux typologies que nous avons identifiées dans cet article. Les évolutions des pédagogies et des pratiques éducatives obligent à repenser ces formes d'un autre temps, pour les adapter aux enjeux actuels et de demain.

Figure 1 : frise chronologique de l'architecture scolaire en France

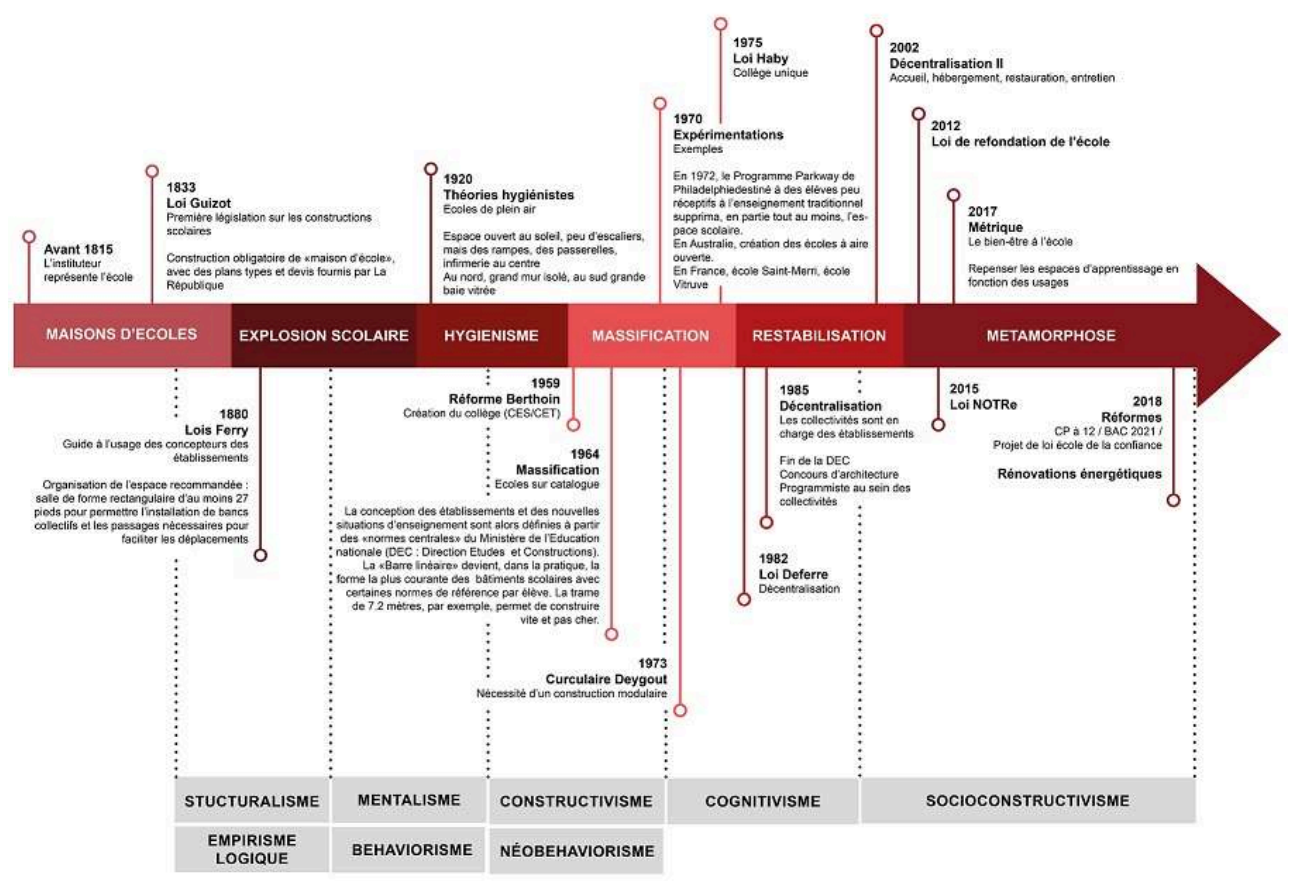


21 Cette relecture de l'histoire de l'architecture scolaire depuis son apparition jusqu'à la fin des années 1970 permet d'établir plusieurs "courants ", c'est-à-dire des grandes tendances constructives en lien avec différents enjeux de société (Figure 1). En premier lieu, l'architecture semble s'être mise au diapason des besoins pédagogiques. C'est le cas pour les bâtiments construits au début du $19^{\text {ème }}$ siècle, les maisons d'école étant liées à l'un ou l'autre des modes (simultané ou mutuel) et dessinées en conséquence. La période de construction sous Jules Ferry lie également étroitement le bâti au mode d'enseignement. La méthode d'enseignement privilégiée est alors le mode simultané ou mode confessionnel (Châtelet, 2008) et les classes sont formées de rangs faisant face au tableau et au professeur.

23 Une seconde période dans l'histoire de l'architecture scolaire, bien que très courte, et peu représentée en France, fait également état d'un lien renforcé entre les pédagogies et l'espace qui les accueillent : les années 1970. Le raisonnement est ici différent de la première période, les expérimentations étant davantage des réactions par rapport aux périodes précédentes, qu'une volonté étatique. D'ailleurs, les expérimentations se font à l'échelle des villes, mais pas au niveau de l'Etat, qui est alors en train de céder la construction et la gestion des bâtiments aux collectivités territoriales (loi Haby, 1975). Plus développée en Suisse, les classes à aires ouvertes seront rapidement recloisonnées après des réactions de la part des enseignants (Foster, 2004).

Une autre tendance, en réaction à la pollution des villes et aux cas de plus en plus nombreux de tuberculose, s'impose comme une réflexion pour le bâti scolaire au début du $20^{\text {ème }}$ siècle : l'hygiénisme. Déjà esquissées avec Jules Ferry, et la mise en œuvre de bâtiments limitant les cas de scoliose et de myopie, les théories hygiénistes vont plus loin, en tentant de repenser l'école dans son entier, depuis le bâti jusqu'aux pratiques (Châtelet et al. 2003). Les expérimentations des écoles de plein air sont relativement peu répandues en France, et définitivement perdues après les années 1930. Toutefois, elles ont le mérite de questionner l'architecture scolaire d'alors, dans sa forme et dans sa temporalité. En effet, les bâtiments sont conçus pour être ouverts sur la nature environnante, voire complètement démontés pour être reconstruits d'une autre manière (Marchoux, 1922). Il en résulte des écoles sous forme de petites maisons disséminées dans la pente herbeuse, toujours orientées pour recevoir un maximum de soleil et d'air.

Enfin, une troisième tendance peut être identifiée, cette fois du fait des urgences économiques. La fin de la guerre et les importantes destructions, couplées à une migration des habitants du monde rural vers la ville, oblige l'État à planifier une intense période de construction d'établissements scolaires (Lainé, 1996). Dans un souci d'économie, de simplicité, d'efficacité et de rapidité, les bâtiments sont construits selon des trames, voire préfabriqués en usine et montés sur place. Ils peuvent accueillir davantage d'élèves que toutes les autres périodes identifiées (on parle de cité scolaire). La rapidité et l'économie de moyens engendre cependant des bâtiments qui peuvent se révéler dangereux pour les usagers, comme l'a montré l'incendie meurtrier de 1973.

Fort de ces constatations, une nouvelle question se pose. Est-on aujourd'hui dans une quatrième tendance concernant l'architecture scolaire? Plusieurs pistes tendent à le suggérer: de plus en plus de recherches se penchent sur le concept de bien-être à l'école (Lippman, 2010 ; Brooks, 2012 ; Barrett et al., 2015 ; Wall, 2016) ; le climat scolaire est en partie relié au bâti, présenté comme l'un des six facteurs déterminants (Debarbieux et al., 2012) ; les rapports de tendances place l'environnement physique au 
cœur des challenges des prochaines années (NMC/CoSN Horizon Report, 2017). La suite de cet article va chercher à mettre en lumière la représentation de l'école et du bienêtre pour la société mais également sous le prisme des architectes.

\section{Architecture scolaire aujourd'hui}

Au cours des dernières années, de nombreux appels d'offre ont été publiés concernant les établissements scolaires. Dans les derniers mois, un échantillon de ces appels d'offre semble s'orienter vers une idée du bien-être à l'école. Bien que le nombre limité de ces appels d'offres ne nous permettent pas d'établir une tendance avérée, leur lecture propose tout de même les prémices d'un nouveau type d'appel d'offres pour les établissements scolaires. Plusieurs de ces appels d'offres se placent dans une logique de service aux usagers (Normandie, Chanteloup, Clichy-sous-Bois) avec les notions d'ouverture, de partage, de mutualisation et d'échanges. Les établissements scolaires sont également davantage présentés comme un espace commun aux équipes éducatives et aux élèves, mais également au reste de la communauté : les habitants (Clichy-sousBois), d'autres élèves, voire des entreprises (Normandie). Le mot «bien-être » est écrit dans l'offre de Normandie, relié au care, et à l'idée d'un esprit sain dans un corps sain.

Dans le même temps, de nombreuses collectivités s'emparent du sujet du bien-être à différentes échelles, au sein des écoles et des établissements scolaires : les oasis de fraicheur dans les cours d'école de la Ville de Paris, la concertation des usagers en Région Normandie ou en Occitanie, le bien-être des personnels de la Ville de Toulouse ou encore les contrats d'assistance à maitrise d'usage qui tendent à se développer.

Parallèlement, de nombreuses études internationales montrent, ces dernières années, l'impact de l'environnement sur la santé et le bien-être des usagers (Lippman, 2010; Brooks, 2012 ; Barrett et al., 2015b). Plusieurs états se sont également emparés du sujet : la Nouvelle-Zélande (Wall, 2016), l'Australie qui pose la question des écoles modulaires (Future Proofing School, 2012) ou encore, plus près de nous, l'Ecosse qui a entamé une refonte de son système dès 2007 (Building Excellence, 2007) menant en parallèle une transformation des pratiques et des bâtiments. Les caractéristiques physiques des établissements scolaires sont ainsi pointées : lumière, acoustique, qualité de l'air. Le rapport britannique "Clever Classrooms", l'une des premières études holistiques réalisées sur plus d'une centaine de classes de primaire, s'attarde également sur des critères complémentaires, moins quantifiables, mais tout aussi importants au regard du bien-être des usagers tels que le lien à la nature, le niveau de stimulation de l'environnement ou encore le sentiment d'appartenance (Barrett et al., 2015b). Une étude de 2018 dans quatre lycées américains indique que le bâtiment a un impact statistiquement significatif sur les niveaux d'engagement des élèves (Scott-Webber et al., 2018).

\section{La représentation de la société}

30 Pour chercher à comprendre les rapports entre école et bien-être, nous avons établi une grille de lecture sur les 50 premières images qui apparaissent dans le moteur de recherche Google $e^{\circledast}$, ainsi que dans le moteur de recherche d'image iStock ${ }^{\circledast}$. Pour quatre recherches de mots-clefs différents, nous avons appliqué une grille de lecture sur la base des critères relevés par Peter Barrett et al. (2015a) comme étant significatifs sur la 
performance et le bien-être des élèves. Ces critères sont, dans l'étude «Clever Classrooms ", au nombre de dix : sept sont considérés comme primaires et trois comme ayant un impact secondaire.

Tableau $\mathrm{n}^{\circ} 1$ : grille d'observables de Barrett et al. (2015)

\begin{tabular}{|l|l|l|}
\hline \multirow{4}{*}{ Naturalness } & Light & Lumière \\
\cline { 2 - 3 } & Air quality & Qualité de l'air intérieur \\
\cline { 2 - 3 } & Temperature & Température \\
\cline { 2 - 3 } & Sound (secondary) & Son (secondaire) \\
\cline { 2 - 3 } & Links to nature (secondary) & Liens à la nature (secondaire) \\
\hline \multirow{4}{*}{ Individualisation } & Flexibility & Flexibilité \\
\cline { 2 - 3 } & Ownership & Appropriation \\
\cline { 2 - 4 } & Connection (secondary) & Connexions (secondaire) \\
\hline \multirow{2}{*}{ Stimulation - Appropriate level of } & Complexity & Complexité \\
\cline { 2 - 4 } & Colour & Couleur \\
\hline
\end{tabular}

31 Les images analysées étant fixes, plusieurs critères ne peuvent être évalués pour cette recherche. Ainsi, nous choisissons de nous concentrer sur cinq critères, listés dans le tableau ci-après. L'étude est faite sur les 200 premières images proposées par Google ${ }^{\circledast}$ et iStock ${ }^{\circledast}$ pour les recherches suivantes :

1. « école bien-être bâtiment », 50 premières images ;

2. " école bien-être classe ", 50 premières images ;

3. « école innovante ", 50 premières images ;

4. « école du futur », 50 premières images ;

Tableau $n^{\circ} 2$ : grille d'analyse des images disponibles sur Google ${ }^{\circledR}$ et iStock ${ }^{\circledR}$

\begin{tabular}{|l|l|}
\hline Critères & Conditions 1 \\
\hline Lumière & Fenêtre visible sur l'image \\
\hline Naturelle & Éclairage visible sur les murs, positionné au plafond \\
\hline Artificielle & \\
\hline Couleur & $\begin{array}{l}\text { Entre une et trois teintes visibles sur l'image, le blanc et le noir ne } \\
\text { constituant pas une couleur }\end{array}$ \\
\hline $\mathbf{1}$ à 3 &
\end{tabular}




\begin{tabular}{|c|c|}
\hline+ de 3 & Plus de trois teintes dominantes visibles sur l'image (sauf blanc et noir) \\
\hline \multicolumn{2}{|l|}{ Gamme } \\
\hline Chaude & Couleurs tirant vers le jaune, orange, rouge, et matières comme le bois \\
\hline Froide & Couleurs tirant sur le bleu, vert \\
\hline \multicolumn{2}{|l|}{ Flexibilité } \\
\hline \multicolumn{2}{|l|}{ Type de mobilier } \\
\hline Classique & Organisation en rangs, tables et chaises en tubes métalliques \\
\hline Ilots & Tables et chaises organisées en ilots de 4 ou plus \\
\hline Mobiles & Chaises, tables ou autre élément de mobilier monté sur roulettes \\
\hline Détente & $\begin{array}{l}\text { Organisation basée sur des poufs, des canapés, des fauteuils, ou d'autres } \\
\text { mobiliers d'aspect confortable }\end{array}$ \\
\hline \multicolumn{2}{|l|}{ Complexité } \\
\hline Espaces de pause & $\begin{array}{l}\text { Petits espaces avec des livres, des bancs, des fauteuils, où les élèves ont l'air } \\
\text { d'être en autonomie }\end{array}$ \\
\hline Couloirs plus larges & $\begin{array}{l}\text { Possibilité de passer à plus de deux, avec des manteaux ou d'autres } \\
\text { installations + pas de couloirs du tout }\end{array}$ \\
\hline $\begin{array}{l}\text { Grands murs pour } \\
\text { accrochage }\end{array}$ & Surface laissée à l'accrochage supérieure à $50 \%$ du mur \\
\hline Espace large & Espace supérieur à l'espace de classe standard (environ $50 \mathrm{~m}^{2}$ ) \\
\hline Espace complexe & $\begin{array}{l}\text { Espace composé de formes de différentes tailles, alcôves, murs, arrondis, } \\
\text { etc. + Plafonds plus ou moins hauts en fonction des espaces. }\end{array}$ \\
\hline \multicolumn{2}{|l|}{ Nature } \\
\hline Vue & Fenêtre à travers laquelle un arbre ou des plantes sont visibles (vert) \\
\hline $\begin{array}{l}\text { Accès à la nature } \\
\text { (physique) }\end{array}$ & $\begin{array}{l}\text { Une porte est visible, donnant sur l'extérieur, ou présence d'arbres dans la } \\
\text { cour }\end{array}$ \\
\hline Plantes & Plantes visibles dans la classe, à l'intérieur des espaces \\
\hline
\end{tabular}

Nous constatons que $60 \%$ des images de Google ${ }^{\circledast}$ pour la première recherche (« école bien-être bâtiment ») sont directement liées au domaine de l'école. Elles sont 36 \% à représenter des bâtiments vus de l'extérieur, 10 \% représentant des vues intérieures et $8 \%$ représentant autre chose que des constructions. Pour cette même recherche, seule $4 \%$ des images iStock $^{\oplus}$ sont en lien avec l'école, et toutes représentent des vues intérieures, avec des gros plans sur de jeunes enfants. 
l'école du bien-être (recherche 1), école innovante (recherche 2), école du futu (recherche 3) du point de vue des constructeurs. Pour ce faire, nous avons fait une recherche sur la plateforme Archdaily, plateforme internationale répertoriant des milliers de bâtiments d'architecture de toutes sortes. Elle est une référence pour les concepteurs, qui l'utilisent le plus souvent comme source d'inspiration ou pour montrer leurs travaux.

40 Cette analyse s'est faite en deux étapes. Dans un premier temps, nous avons analysé l'image de présentation d'un article répertoriant 70 exemples d'architecture scolaire. Sur cette image, deux types de représentations techniques d'un bâtiment sont proposées : des plans et des coupes. On retrouve ainsi 12 plans et 24 coupes. Aucune information ne nous permet de dater ces dessins, de même que de les lier les uns aux autres. Toutefois, cette image est intéressante dans la variété de formes, de hauteurs et de traitements du sujet qu'elle propose. Sur les 12 exemples proposés, pas un seul n'est similaire. 
41 La deuxième étape a été d'analyser plus en détail les 10 premiers résultats de cet article. Pour analyser les bâtiments, nous avons repris la grille de lecture présentée plus haut, en y ajoutant une composante concernant les matériaux (présence de bois, de minéral ou de métal à plus de $50 \%$ sur les images). Les outils à notre disposition sont des photos de l'ouvrage terminé (des vues intérieures et extérieures), des plans et des coupes techniques.

Figure 2. Architecture scolaire innovante, point de vue des architectes, Archdaily, 2018
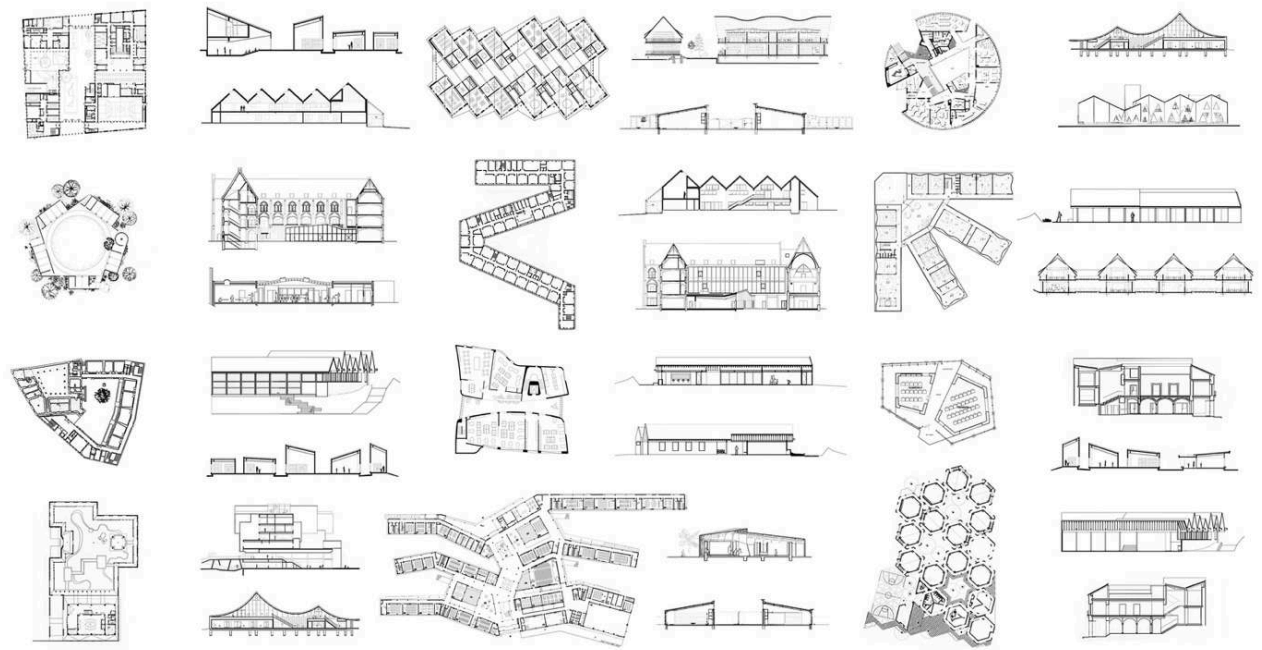

Sur les dix premiers bâtiments, cinq sont localisés en Europe, dont un seul en France, un aux États-Unis, et quatre en Asie. Sept bâtiments sont dédiés au premier degré (écoles primaire), deux au second degré (un collège et un lycée) et un à l'éducation supérieure (école des arts).

Après analyse, nous constatons que $100 \%$ des dix premiers bâtiments ont un apport important de lumière naturelle, et $50 \%$ ont un éclairage artificiel zénithal identifié. Seuls $40 \%$ possèdent une dominance de couleur autres que le blanc, le gris et le noir. Un seul est jaune. $60 \%$ des bâtiments sont à dominante de bois, à l'intérieur aussi bien qu'à l'extérieur. $70 \%$ sont en béton et $40 \%$ en acier. $60 \%$ des bâtiments allient plusieurs matériaux (bois-minéral ou minéral-métal).

En ce qui concerne les dispositifs intérieurs, $40 \%$ des projets présentés sont constitués de mobilier en ilots, $20 \%$ ont des mobiliers classiques, et $20 \%$ des mobiliers de type pouf ou fauteuil. Les $20 \%$ restant ne présentaient pas d'image permettant d'analyser ce critère.

En termes de complexité, $80 \%$ des bâtiments analysés proposent des espaces plus larges que les standards français (environ $50 \mathrm{~m}^{2}$ ), et $70 \%$ présentent une complexité dans leur architecture (escaliers qui deviennent des gradins, balcons, petites et grandes hauteurs sous plafond, etc.). $40 \%$ ont des couloirs plus larges que les standards français et $20 \%$ ont des murs suffisamment grands et vides pour accueillir des affichages. Enfin, $40 \%$ proposent des espaces de détente parfois attenants aux salles principales.

Concernant le dernier critère, le lien à la nature, on constate que $90 \%$ des exemples analysés ont une vue sur la nature (arbre, pelouse ou forêt). $40 \%$ y ont accès depuis les salles de cours ou les espaces principaux et seulement $10 \%$ présentent des plantes à l'intérieur du bâtiment. 


\section{Conclusion}

Prendre en compte le bonheur et le bien-être à apprendre ont été des facteurs nécessaires mais pas suffisants pour engager des transformations majeures des architectures scolaires, d'autres variables étaient en jeu. La recommandation de 1973, testée par des travaux de recherche contemporains est une préoccupation d'actualité pour les acteurs de l'école (collectivité, enseignants, fédération de parents) quand il est question de rénover, réhabiliter ou construire. La collectivité qui voit sa capacité financière réduite et qui cherche à obtenir la bâtiment "parfait» qui peut sans difficulté majeure suivre les besoins en pratiques pédagogiques et en besoins sociétaux, et pour les pédagogues, qui veulent pouvoir avoir des espaces ne les empêchant pas d'agir, source de stresseur environnemental. Dans les années 70, ce sont probablement des contraintes de société, de par la loi Haby, qui a conduit à produire en masse, sans prendre toute la mesure des recommandations faites quelques années auparavant. Estce qu'un virage similaire aura lieu? ou est-ce que la considération du bien-être à apprendre va s'ancrer dans les pratiques ? et de quelles pratiques parle-t-on : au niveau des collectivités, des enseignants, de l'inspection, des élèves, des modèles de financement qui permettront d'avoir un bâtiment dont les équipes pourront habiter les lieux et se les approprier sur plusieurs cycles de vie ? Sommes-nous à la genèse d'un nouveau courant architectural? alors que nous voyons une telle distorsion entre les images rendus disponibles au grand public (Google ${ }^{\circledast}$ et IStock $^{\circledast}$ ) et celles entre spécialistes des espaces construits (Archdaily).

La recherche a encore devant elle un important champ d'études autour du bâtiment scolaire et de son impact sur les pédagogies et les pratiques des usagers. D'un point de vue plus pratique, les nombreuses maitrises d'usage qui s'attachent à accompagner les usagers dans la définition de leurs besoins, se heurtent le plus souvent sur les représentations non partagées des usagers, des maitrises d'ouvrage (collectivités) et des maitrises d'œuvre (architectes, designers, etc.) autour du bâtiment scolaire, dans sa forme physique et dans ses temporalités. Travailler à l'identification et à l'élaboration de visions partagées, et ancrées dans les pratiques d'aujourd'hui et de demain, constitue un terrain fertile pour favoriser le bien-être des élèves et des usagers de l'école en général. Ce travail doit à la fois s'appuyer sur les expérimentations isolées, politiques ou éducatives, ainsi que sur une vision plus systémique de la recherche.

\section{BIBLIOGRAPHIE}

Allen, J. G., et al, (2016). Associations of Cognitive Function Scores with Carbon Dioxide, Ventilation, and Volatile Organic Compound Exposures in Office: A Controlled Exposure Study of Green and Conventional Office Environments. Environment Health Perspectives, DOI: 10.1289/ehp. 1510037

Barrett, P., \& al. (2015a). Clever Classrooms, Summary report of the HEAD Project (Holistic Evidence And Design), University of Salford, February 2015 
Barrett, P., \& al. (2015b). The impact of classroom design on pupils' learning : final results of a holistic, multi-level analysis. Building and Environment, 89, 118-133 [http://dx.doi.org/10.1016/ j.buildenv.2015.02.013]

Brooks, C., (2012). Space and Consequences: The Impact of Different Formal Learning Spaces on Instructor and Student Behavior. Journal of Learning Spaces, 1(2)

Chatelet, A.-M. (1999). La naissance de l'architecture scolaire, les écoles élémentaires parisiennes de 1870 à 1914. Paris : Honoré Champion

Chatelet, A.-M. (2008-2009). « Deux siècles de bâtiments scolaires en France (XIXe-XXe), Cours publics, Cité de l'architecture et du patrimoine »-Conférence en ligne

Châtelet A.-M. \& Lerch D, Luc J-N (dir.) (2003). L'école de plein air. Une expérience pédagogique et architecturale dans l'Europe du XXe siècle. Paris : Éditions Recherches, 431 p.

Debarbieux, E., Anton, N., Astor, R.A., Benbenishty, R., Bisson-Vaivre, C., Cohen, J., Giordan, A., Hugonnier, B., Neulat, N., Ortega Ruiz, R., Saltet, J., Veltcheff, C., Vrand, R. (2012). Le « Climat scolaire » : définition, effets et conditions d'amélioration. Rapport au Comité scientifique de la Direction de l'enseignement scolaire, Ministère de l'éducation nationale. MEN-DGESCO/Observatoire International de la Violence à l'École. 25 pages.

Daly, J., Burchett, M., \& Torpy, F., (2010) Plants in the classroom can improve student performance, Report to IPA (Australia)

Derouet-Besson M.-C., (2005). Le patrimoine architectural : l'apport de l'école à la construction d'une culture architecturale en France ». Revue de l'inspection générale : Construire pour enseigner, p4-20.

Deoux, S. (2010). Bâtir pour la santé des enfants. Paris : Medieco Editions

Durandet, D. (2014). L'école d'antan en 300 images. Paris : Massin

Felguerias Margarida L., 2007. «A arquitectura da escola primaria em Portugal nos séculos XIX e $\mathrm{XX} »$. In La escuela y sus escenarios. El Puerto de Santa Maria, p 31-65.

Fischer, G. (2011). Psychologie sociale de l'environnement. Paris : Dunod

Forster, S. (2004). Architecture scolaire : regard historique tourné vers l'avenir. L'architecture scolaire, Bulletin de la CIIP (15), 3-9

Gauthier, M. (1911). Les écoles de plein-air, une étape dans la lutte sociale anti-tuberculeuse. Thèse présentée à la Faculté de Médecine.

Hall, E.T. (1966). La dimension cachée. Paris : Seuil.

Jeannin, L. (2017). La mobilité, clé de nouvelles pratiques ?. Education et société, 43

Jetsonen S. \& Johansson E., 2011. The best School in the World: Seven Finnish Examples from the 21st Century. Helsinki: Musuem of Finnish Architecture, 80 p.

Knittel, F. \& Castets-Fontaine, B. (2015). Le système scolaire en France du XIXème siècle à nos jours. Paris: Ellipses.

Karwowski, W. (2005). Ergonomics and human factors: the paradigms for science, engineering, design, technology and management of human-compatible systems. Ergonomics, 48(5), 436-463.

Lainé, M. (1996). Les constructions scolaires en France. Paris : PUF. 
Le Cœur, M. (2005). L'architecture et l'installation matérielle des lycées. La réglementation et sa mise en œuvre (1802-1940). In : Lycées, lycéens, lycéennes, deux siècles d'histoire. Paris : Institut national de recherche pédagogique. pp. 363-380. (Bibliothèque de l'Histoire de l'Education, 28)

Lippman, P.C. (2010). Can the physical environment have an impact on the learning environment?. CELE Exchange 2010/2013, OECD

Marchand, B. (2004). L'architecture scolaire aujourd'hui. Bulletin CIIP, n 15, décembre, p20-23.

Marchoux, Pr. (1922). L'air à l'École. Hygiène par l'exemple, $n^{\circ} 1$

Martinho M., José M. \& R. Freire da Silva, 2008. Portugal - Les écoles bâties sur le modèle de l'espace paysager : échec ou innovation ?, OCDE, CLE : https://www.oecd.org/fr/portugal/ 41533189.pdf

Mazalto M., Bonnault M.-C. \& Boudjémaï Z. (2008). Architecture scolaire et réussite éducative. Paris : Fabert.

Mozaffar, F., \& Somayeh Mirmoradi, S. (2012). Effective Use of Nature in Educational Spaces Design. Organization, Technology \& Management in Construction: An International Journal, 4(1), 381-392.

Navarro, M. (2012). « Massification de l'enseignement, inégalités d'apprentissage et pratiques pédagogiques ", Regards croisés sur l'économie 2012/2 ( $\left.{ }^{\circ} 12\right)$,

p. 203-207.

Poignant R. (1960). La planification de l'expansion de l'enseignement en France. In : Tiers-Monde, tome $1, \mathrm{n}^{\circ} 1-2$. La planification de l'éducation et ses facteurs économiques et sociaux. Colloque international de Paris (9-18 décembre 1959) pp. 208-220

Prost A. (2005). Jalons pour une histoire de la construction des lycées et collèges de 1960 à 1985. In : Lycées, lycéens, lycéennes, deux siècles d'histoire. Paris : Institut national de recherche pédagogique. pp. 459-478. (Bibliothèque de l'Histoire de l'Education, 28)

Prost, A. (2007). Regards historiques sur l'éducation en France : XIXe-XXe siècles. Paris : Belin.

Rascle, N., \& Bergugnat, L. (2016). Qualité de vie des enseignants en relation avec celle des élèves : revue de question, recommandations. Contribution dans le cadre du rapport du Cnesco sur la qualité de vie à l'école.

Schleifer S. (dir.), (2008). Crèches, écoles et lycées. Paris : Place des victoires.

Selye, H. (1946). The general adaptation syndrome and the diseases of adaptation. The journal of clinical endocrinology, 6(2), 117-230.

Scott-Webber, L. (2018). Hack my space, cueing behaviors // Aligning Teaching Strategies with Building Design.

Tenailleau, N. (2018). L'école ouverte : un espace pour la mixité, Dossier $n^{\circ} 487$ «Filles et garçons à l'école », Cahiers Pédagogiques, Novembre 2018.

Torres, V., Sanders, M. \& Corsi, R. (2002). Texas elementary school indoor air study (TESIAS) : overview and major findings. Indoor Air

Wall, G. (2016). Report: The impact of physical design on student outcomes. Ministry of Education, New Zealand.

Weinstein, C. S. (1992). Designing the Instructional Environment: Focus on Seating. 
Willard Magaud, N. (1973), De la classe ouverte à l'aire ouverte, Education et développement $\mathrm{n}^{\circ} 86$, mai-juin 1973.

\section{RÉSUMÉS}

Au cours de l'histoire de l'architecture scolaire, plusieurs tendances se sont dégagées, le plus souvent en rapport avec les enjeux de la société de l'époque. Parmi ces tendances, on retrouve les maisons écoles, l'explosion scolaire, l'hygiénisme, la massification, la restabilisation, et l'actuelle métamorphose du parc foncier. Cet article fait l'hypothèse que la société actuelle entre dans un questionnement à plusieurs variables entre une gestion financière et de l'espace foncier occupé plus fine, une nécessité de modularité gage d'adaptation sur le long terme, et les conditions d'exercice corrélés aux conditions de bien-être et du bonheur à apprendre. Pour travailler cette question, nous nous essayons à une frise chronologique entre étapes architecturales, pédagogie dominante ou réponse remarquée à une pédagogie dominante, et contexte politique. Enfin, au travers de site spécialisé en architecture, nous analysons ce qui est donné à voir comme architecture scolaire remarquable.

During the history of school architecture, several trends have emerged, most often in relation to the challenges of the society of the period. These trends include school houses, the school explosion, hygiene, massification, restoration and the current metamorphosis of the land base. This article hypothesizes that today's society faces a questioning of several variables, between a management of finances and the land space occupied, a need for modularity as a guarantee of long-term adaptation, and conditions of exercise correlated to conditions of well-being and happiness to learn. To work on this question, we try to create a chronological framework between architectural stages, dominant pedagogy - or a noticeable response to a dominant pedagogy - and political context. Finally, through sites specializing in architecture, we analyze what can be seen as outstanding school architecture.

\section{INDEX}

Keywords : school architecture, dominant pedagogy, particular, well-being, happiness to learn Mots-clés : architecture scolaire, pédagogie dominante, particulière, bien-être, bonheur à apprendre

\section{AUTEURS}

\section{SARAH BARTHELEMY}

Université de Cergy-Pontoise, membre de la chaire de recherche Transition2

\section{LAURENT JEANNIN}

Université de Cergy-Pontoise, MCF, laboratoire BONHEURS (EA 7517), titulaire de la chaire de recherche Transition 2 : des espaces en transition à la transition des espaces 